\title{
Le rôle de l'enseignant en situations de débat sur des thématiques de l'éducation en vue du développement durable
}

\section{Nathalie Freudiger et Philippe Haeberli}

Ce texte sinscrit dans une recherche menée dans des écoles de Suisse romande par l'Équipe de recherche en didactiques et épistémologie des sciences sociales (ERDESS) de l'Université de Genève. Il traite spécifiquement de la place et du rôle de l'enseignant en situations de débats; les auteurs sintéressent, dans ces situations, au poids des habitudes et des contraintes scolaires, non seulement du point de vue de la dynamique des échanges, mais également du point de vue du contenu des propos échangés. Les analyses de quatre débats menés dans deux classes, l'une primaire, l'autre secondaire, montrent que les situations de débat n'échappent pas aux tensions coutumières qui caractérisent l'enseignement scolaire. Le rôle de l'enseignant est analysé notamment à l'aide d'une triple distinction relative aux fonctions de régulation de l'échange qu'il est amené à endosser.

Reposant sur une recherche menée en éducation en vue du développement durable dans des écoles de Suisse romande, ce texte vise à saisir les aspects didactiques de situations de débat à propos de questions socialement vives ${ }^{1}$. Notre contribution aborde plus spécifiquement le rôle et la place de l'enseignant en situation de débat et fait appel aux notions de coutume et d'étayage pour qualifier les tensions auxquelles les enseignants sont confrontés dans ces situations. Nous soulignons le poids des habitudes et des contraintes scolaires non seulement du point de vue de la dynamique des débats mais également du point de vue thématique et du contenu des propos échangés.

La présente contribution s'appuie sur le premier volet achevé ${ }^{2}$ d'une recherche intitulée «Les contributions des enseignements de sciences sociales - histoire, géographie, citoyenneté - à l'éducation en vue du développement durable. Étude d'un exemple: le débat en situation scolaire»; la recherche, financée par le Fonds national suisse de la recherche scientifique, est menée par l'Équipe de recherche en didactiques et en épistémologie des sciences sociales (ERDESS 3 ).

Le texte est divisé en deux parties. La première partie est dédiée à la présentation de l'enquête et des notions théoriques; la seconde présente des résultats à partir d'analyses menées dans deux classes, l'une de primaire, l'autre de secondaire. 


\section{Présentation de la recherche, des notions de coutumes didactiques et de la fonction de régulation de l'échange}

\section{La recherche}

Dans le sillage du développement durable qui s'est imposé à l'action politique depuis le rapport de la Commission Brundtland en 1987, des déclarations et des initiatives sociales et politiques se multiplient en faveur d'une éducation en vue du développement durable. Cependant, l'insertion de cette éducation dans les plans d'études européens n'est pas évidente, puisqu'il ne s'agit pas d'une nouvelle discipline, mais qu'à l'instar d'autres "éducations à...", l'éducation en vue du développement durable doit privilégier des approches interdisciplinaires. Or, les contenus et conditions de l'interdisciplinarité restent encore très largement à explorer (Lenoir \& Sauvé, 1998). Celle-ci n'est pas un concept, encore moins une pratique, qu'il suffirait d'énoncer et de suggérer pour qu'elle soit construite et mise en œuvre de façon rigoureuse. Par ailleurs, l'École et les plans d'études (dont, en Suisse, le futur Plan d'études romand en cours d'élaboration) sont conçus à partir de la différenciation des disciplines.

Compte tenu de ses enjeux, des défis qu'il pose, voire de ses ambiguïtés, le développement durable appelle des solutions qui sont avant tout politiques, car elles mettent en jeu les intérêts, les rapports de force, les croyances, les attentes, entre les individus et les groupes, les États, les entreprises, les associations, etc. Les sciences sociales sont mobilisées pour construire des outils, des savoirs, des points de vue, des compétences, permettant de mieux raisonner ces solutions et de contribuer au débat et aux choix politiques. Ainsi, l'éducation en vue du développement durable requiert nécessairement des savoirs de sciences sociales, savoirs présents dans la plupart des curriculums scolaires de l'école obligatoire principalement à travers l'histoire, la géographie et l'éducation à la citoyenneté. Pour construire ces savoirs et compétences, le projet de recherche est inscrit dans la problématique «du détour et du retour». Au départ, il y a une réalité sociale qui appelle actions et décisions. Cette réalité n'est pas disciplinaire, les décisions à prendre non plus. Mais l'étudier et l'analyser requiert des outils et des savoirs disciplinaires. C'est la phase du détour au cours de laquelle sont introduits et construits des savoirs et des outils disciplinaires. Puis il s'agit de mobiliser ces savoirs dans une situation différente, c'est la phase du retour. Pour tenter de saisir dans le moment de retour ce que les interactions entre protagonistes de la classe produisent, deux situations de débats ont été mises en œuvre dans le cadre d'une séquence didactique.

Les deux situations de débats prennent place après un moment d'étude d'une question relative au développement durable. Toutes les classes ont travaillé sur deux exemples caractérisés par un accident climatique extrême ayant eu des conséquences humaines d'intensité différente selon le contexte: les inondations à un 
niveau local en Suisse, durant l'été 2005 à Brienz-Glyssibach (BE), durant l'été 2007 à Roche (VD) ou Flamatt-Wünnewil (FR) et la mousson particulièrement intense qu'a connue le Bangladesh durant ce même été 2007.

Le premier débat (D1) est un débat de type consensuel et concerne la situation en Suisse. Il a pour but de demander à une autorité politique de prendre deux mesures importantes pour éviter que de tels événements se reproduisent. Quatre phases organisent initialement celui-ci:

1) la présentation du débat, de son thème et de son enjeu;

2) la formulation de propositions de mesures par les élèves;

3) la justification par les élèves des deux mesures que chacun considère être les plus importantes;

4) le choix des deux mesures à proposer.

Le second débat (D2) est un débat de type contradictoire: il a pour but de mettre les élèves en situation de convaincre les autres. L'enjeu est de répartir une somme virtuelle de 1000 francs entre quatre projets ${ }^{4}$, destinés à faire face aux changements climatiques et leurs conséquences au Bangladesh, en Suisse ou à l'échelle planétaire. Il est constitué des phases suivantes: individuellement, les élèves doivent décider du partage de la somme entre les quatre projets. Ensuite ils débattent collectivement en argumentant pour défendre leur choix. Et enfin, après avoir entendu les différents arguments, les élèves signalent s'ils ont changé d'avis par rapport à leur premier choix.

\section{Le débat, un pli dans la coutume}

Dans le monde francophone, les travaux en didactiques menés depuis une trentaine d'années visent à saisir ce qui définit le cadre de vie ou de pensée des élèves et des enseignants lorsqu'ils font leur métier d'élève ou de professeur. La notion de contrat a été l'une des notions qui a connu le plus de succès pour analyser les relations entre maître et élèves. Pour l'histoire et la géographie scolaires, Audigier (1991) a montré la force du contrat disciplinaire, qui se traduit par un jeu d'attentes dans les enseignements de ces disciplines. Ces attentes s'emploient à construire des conceptions que les acteurs se font des rôles respectifs qu'ils doivent tenir dans le cadre des cours liés à ces enseignements disciplinaires; ces conceptions laissent, en général, peu de place à des moments de travail et de réflexion ou sont même des obstacles pour de tels moments. Dans une étude comparative de cinq leçons d'histoire à l'école primaire en Suisse et en France, il est montré que le jeu des questions/réponses mené sur un rythme très rapide et dominant très largement la dynamique des leçons amène les enseignants à faire des simplifications qui deviennent de possibles obstacles didactiques (Audigier, 1998; Audigier, Auckenthaler, Fink \& Haeberli, 2002). Par exemple, l'assimilation et l'attribution de propriétés communes à des catégories de personnes (en l'occurrence, personnes vivant à la campagne à paysans) interrogent la compréhension de la place des acteurs dans les réalités passées et présentes. Ainsi, en situation d'enseignement scolaire, la recherche d'un consensus autour de «ce qui doit être su» prime 
le plus souvent sur l'entrée dans la complexité du monde; le référent consensuel, rarement questionné, prend par ailleurs une forme tacite et implicite.

Pour interpréter ces phénomènes, Balacheff (1988) propose de les placer dans le temps long de l'enseignement, dans le cadre de «règles de fonctionnement social de la classe», des coutumes didactiques ou scolaires. Par ce dernier terme, Balacheff désigne plus spécifiquement la dimension sociologique du contrat disciplinaire et sa perméabilité des savoirs à la dimension sociale des relations dans la classe. Il montre en particulier, à partir d'un exemple de débats en mathématique, que l'explicitation des règles déterminant le type de relations sociales de la classe a un impact sur la construction des savoirs.

La classe est une société coutumière. Nous entendons par coutume un ensemble de pratiques obligatoires, de façons d'agir établies par l'usage; le plus souvent implicitement. La coutume règle la façon dont le groupe social entend que soient établis les rapports sociaux (...). L'explicitation des règles change la nature des interactions sociales dans la classe. Passage d'une société coutumière à une société de droit, cette transformation est autre chose qu'une simple modification technique, elle modifie le comportement et donc la signification des savoirs construits dans le nouveau cadre. (Balacheff, 1988, pp. 21-23).

Certaines coutumes scolaires sont bien établies et se retrouvent dans toutes les salles de classe; la manière dont la circulation de la parole est organisée est probablement l'exemple de coutume la mieux établie dans les écoles ${ }^{5}$. Mais il existe également une série de règles coutumières propres aux contextes d'enseignement, autrement dit à la discipline enseignée ainsi qu'aux pratiques enseignantes. L'une des hypothèses que nous illustrons plus loin est que le débat en classe met en lumière les tensions coutumières qui caractérisent l'enseignement scolaire. Situation autorisant et encourageant les échanges et les interactions entre les élèves eux-mêmes et non plus seulement entre le maître et les élèves pris comme un groupe, la situation de débat opère un élargissement ainsi qu'une complexification certaine de la configuration de base "maître-élèves» dans les mécanismes de construction du savoir 6 .

\section{Contenu du discours et régulation des échanges}

Le rôle central de l'oral dans les apprentissages scolaires, la nécessité pour l'École d'assurer un réel apprentissage de la prise de parole font depuis quelques années l'objet de nombreux discours dans le champ institutionnel comme dans celui de la recherche en didactique (Dolz \& Schneuwly, 1998), en sciences du langage et en psychologie de l'apprentissage. Parmi les courants de la recherche, nous privilégions l'axe proposé par Elisabeth Nonnon (1997); cette dernière confronte deux dimensions réputées irréductibles à une analyse rigoureuse, l'analyse des objets du discours d'une part, la temporalité dans laquelle celui-ci s'inscrit de l'autre. Nous postulons ainsi avec Grize (1996) que la richesse d'une interaction sociale tient, en particulier, à la façon dont ce dont on parle, les «objets de discours» et les «schématisations» y prennent consistance, se complexifient, se réorganisent. 
En effet, selon Grize, le discours met en œuvre tout un ensemble d'activités qui font émerger certains objets; autrement dit, les propos sur le monde découpent, qualifient celui-ci, ils le chargent également de telle valeur, l'appréhendent de tel ou tel point de vue, ou encore mettent en relation des objets entre eux, établissent des comparaisons. Ainsi, un travail sur les «objets du discours» implique d'identifier les opérations discursives et les processus qui, en permanence, réorganisent les objets du discours; parmi celles-ci, les reformulations, les déplacements thématiques, les changements d'éclairage, de modalité ou de catégorisation, etc. L'évolution de la signification nécessite la prise en compte de la temporalité des échanges, du positionnement dynamique des énoncés ou des arguments les uns par rapport aux autres, de la succession des moments thématiques, des unités argumentatives, etc.

Selon Bruner (1983/1996), l'étayage est un processus

"[...] qui rend l'enfant ou le novice capable de résoudre un problème, de mener à bien une tâche ou d'atteindre un but qui auraient été, sans cette assistance, audelà de ses possibilités. [...] Il peut, pour finir, produire un développement de la compétence de l'apprenti pour cette tâche à un rythme qui dépasse de beaucoup celui quil aurait atteint par ses efforts sills étaient restés sans aide» (p. 263).

S'appuyant sur Grize et Bruner (1996), Nonnon (1997, p. 36) distingue trois fonctions des régulations de l'échange facilitant les tâches discursives scolaires liées à l'appropriation des connaissances et à la construction du jugement:

1. la fonction de finalisation. Les régulations de l'échange orientent les énoncés par rapport à une visée, «en explicitant ce vers quoi ils tendent et en maintenant la tension de l'échange par rapport à une question»;

2. la fonction de développement. Les régulations de l'échange appellent à poursuivre «l'expansion d'un développement thématique, et d'un développement suscitant les dénivellations d'un discours»;

3. la fonction d'intégration et de séquentialisation. Les régulations de l'échange délimitent «les frontières des unités thématiques par diverses formes de bouclage et de clôture en donnant une cohérence rétrospective à ce qui vient d'être dit, par négociation de l'initiative thématique».

Notons que ces fonctions ne sont pas l'apanage de celui qui anime le débat. Tous les participants à un débat, et, dans le cas du débat en classe, tous les élèves sont, en principe, en mesure de mettre en ouvre ces fonctions au cours des échanges. Nonnon montre notamment que certains élèves synthétisent des propos parallèles précédemment énoncés, explicitent une mise en relation de divers moments de l'échange, formulent une question amenant une ouverture thématique, etc. 


\section{Le rôle de L'enseignant et la place des élèves, analysés dans deux classes}

L'analyse de quatre débats dans deux classes que nous proposons porte sur le rôle et la place de l'enseignant dans la régulation de l'échange; en creux, il s'agit également de questionner la place des élèves dans les débats. Dans une grande majorité des débats observés, l'enseignant joue le rôle de l'animateur du débat; toutefois, dans un des débats retenus, un élève est chargé de ce rôle. Notre questionnement s'articule autour de deux dimensions: la dynamique et le contenu des débats. Pour la première, nous nous sommes intéressés à la question spécifique de la circulation et de la prise la parole. Comment la circulation entre les protagonistes s'organise-t-elle? Qui prend la parole durant les débats? Avec quelle fréquence? Quel poids la parole de chacun, et notamment celle de l'enseignant, a-t-elle sur l'ensemble? Telles sont les questions qui ont orienté les analyses. Pour la dimension de contenu, nous avons pris en compte plus particulièrement les aspects de réception et de validation des propos des élèves par l'animateur du débat, l'orientation et la relance des thèmes de discussion, la mobilisation et l'appel à des savoirs, rencontrés ou non dans les moments de travail. Pour ce faire nous réinvestissons, à notre manière, les outils proposés par Nonnon (1997) et évoqués plus haut.

Pour rendre une certaine justice à la diversité des niveaux scolaires et des contextes cantonaux des sept classes ayant participé à l'enquête, nous avons choisi d'analyser deux classes provenant de cantons différents et issus de niveaux d'enseignement distincts. L'une des classes (C1) est une sixième primaire, dernier degré du primaire dans le système scolaire concerné; l'autre classe $(\mathrm{C} 2)$ est une classe de neuvième année (de niveau $\mathrm{B}$, relativement faible), dernière année de l'école obligatoire. La présentation des résultats se divise en trois parties distinctes. Une première partie regroupe et compare les quatre débats sous l'angle de la circulation et de la prise de parole des protagonistes; elle repose sur les comptages des interventions ${ }^{7}$ ainsi que sur la moyenne des mots prononcés ${ }^{8}$. La deuxième partie présente les analyses des débats de la classe primaire en décrivant, successivement, ce qui relève de l'intégration et de la séquentialisation, ce qui peut être compris comme de la finalisation dans les propos de l'enseignant pour s'intéresser, dans un troisième temps, aux effets de la reformulation. La troisième partie, qui présente les analyses pour la classe secondaire, s'appuie non pas sur un découpage thématique, mais sur l'analyse spécifique d'un extrait jugé particulièrement significatif du rôle de l'enseignant dans les débats.

\section{Comptages des interventions et des mots prononcés}

Dans le tableau ci-dessous, nous indiquons le résultat des comptages effectués pour chaque débat (D1 et D29) dans chacune des deux classes (C1 et C2). Quatre types de résultats y sont présentés; ils concernent deux acteurs, l'animateur et les protagonistes ${ }^{10}$. En ce qui concerne le premier, le poids de ses interventions 
par rapport à la somme totale des interventions durant le débat ${ }^{11}$ ainsi que la moyenne des mots prononcés par ce même animateur durant chaque intervention sont indiqués. Pour les protagonistes, le nombre d'entre eux ayant pris la parole durant le débat ainsi que la moyenne de mots prononcés par tour de parole sont indiqués.

Tableau 1: Comptages des interventions et des mots prononcés durant les débats.

\begin{tabular}{|c|c|c|c|c|}
\hline Classe_débat & $\begin{array}{c}\text { Interventions de } \\
\text { l'animateur en \% } \\
\text { du total des } \\
\text { interventions }\end{array}$ & $\begin{array}{c}\text { Mots prononcés } \\
\text { par l'animateur } \\
\text { (moyenne par } \\
\text { intervention) }\end{array}$ & $\begin{array}{c}\text { Nombre de } \\
\text { protagonistes }\end{array}$ & $\begin{array}{c}\text { Mots prononcés } \\
\text { par les } \\
\text { protagonistes } \\
\text { (moyenne par } \\
\text { intervention) }\end{array}$ \\
\hline C1_D1 & $21 \%$ & 22 & 16 & 27 \\
\hline C1_D2 & $44 \%$ & 20 & 18 & 24 \\
\hline C2_D1 & $63 \%$ & 36 & 10 & 20 \\
\hline C2_D2 & $44 \%$ & 5 & 20 & 29 \\
\hline
\end{tabular}

Malgré une certaine hétérogénéité, les animateurs des débats interviennent fréquemment dans l'ensemble des débats: dans les débats C1_D2, C2_D1, et C2_D2, les fréquences sont très élevées. Par ailleurs, dans les débats de la classe $\mathrm{C} 1$, les interventions de l'animateur et des protagonistes sont à peu près de la même longueur. En revanche, dans le premier débat de la classe C2, les interventions de l'enseignant sont nettement plus longues que dans le deuxième débat de cette même classe. Ainsi, ces résultats indiquent que dans les débats analysés, la parole de l'enseignant est quantitativement plus importante que celle des élèves: il intervient beaucoup plus souvent que les élèves, même si ses prises de parole ne sont pas, ou pas beaucoup, plus longues que celles des élèves.

Enfin, le débat C2_D2 présente une particularité: l'animation est assurée par un des élèves de la classe, alors que celle-ci est assurée par l'enseignant dans les trois autres débats. La moyenne des mots prononcés par l'animateur y est très largement inférieure à celle des autres débats alors que la moyenne des mots prononcés par les protagonistes y est la plus élevée des quatre débats ${ }^{12}$.

La comparaison des interventions entre le premier et le deuxième débat de la classe secondaire montre que si l'élève animateur intervient relativement fréquemment, il le fait de manière moins fréquente que l'enseignant. Surtout, si l'enseignant a une moyenne de 36 mots par intervention, celle-ci est de 5 mots seulement pour l'élève animateur. Même si ce dernier n'a pas eu la tâche de présenter le débat, cette moyenne montre un poids bien moindre de la parole de l'animateur élève dans les débats. Elle a aussi des effets notoires sur la participation et la prise de parole. Si dans le débat mené par l'enseignant, seuls dix élèves s'expriment, ils sont une vingtaine à prendre la parole dans le débat mené par l'élève. Ce phénomène ne s'observe pas dans la classe de primaire $(\mathrm{C} 1)$. 


\section{Analyse spécifique des débats de la classe de primaire Fonction d'intégration et de séquentialisation}

Dans les deux débats de la classe primaire analysés dans la présente contribution, il apparaît que l'étayage de l'enseignant (ci-après $M$ ) remplit une fonction d'intégration et de séquentialisation tout au long du débat.

Dans le premier débat, au début de la séquence, $M$ ouvre la discussion de la manière suivante:

(10) ${ }^{13}$ Alors, ce que vous allez faire, c'est donc faire vos propositions dans un premier temps. Votre maîtresse les écrira au tableau noir et ensuite, il faudra que l'on choisisse les deux trois meilleures propositions. Donc, je rappelle que vous n'êtes pas du tout évalués, vous y allez, vous donnez vos propositions, vous n'hésitez pas, et attendez bien d'avoir le micro pour parler. Voilà. Qui veut commencer? Il y a beaucoup de mains déjà levées.

Plus loin, $M$ intervient pour stopper l'énonciation des propositions par les élèves et pour passer à une nouvelle étape, celle du débat sur les propositions. La séquence vidéo semble montrer que $M$ interrompt un moment d'échange entre les élèves :

(98) M: Bien, maintenant, je vous rappelle que nous devons choisir deux propositions. Alors, prenez juste un petit moment pour voir celles qui sont écrites au tableau. Vous arrivez bien à les lire? Et choisissez-en deux, personnellement, chacun, deux qui vous semblent les meilleures. Et il faudra essayer de se décider sur les deux meilleures. Donc convaincre ceux qui ne pensent pas que ce sont les deux meilleures les vôtres, de prendre votre avis. Donc, lisez d'abord bien les huit. Neuf, propositions.

$M$ tient également le déroulement du deuxième débat entre ses mains. Il annonce le commencement du moment où les élèves doivent partager la somme de 1000 francs entre les quatre projets de la manière suivante:

(164) Alors qui veut commencer? qui veut donner sa position?

Après que les élèves ont argumenté pour justifier leur partage de la somme, une élève intervient et dit:

(252) Ben je sais pas moi, j'ai... heu...avec les conseils des autres j'ai un peu changé d'avis.

On peut poser l'hypothèse que cette intervention aurait pu engendrer le passage à une nouvelle étape de la séquence, celle lors de laquelle les élèves doivent se demander s'ils ont changé d'avis depuis le début du débat. Mais $M$ répond de la manière suivante à l'élève:

(253) D'accord. Mais dis ce que tu avais noté d'abord. Parce qu'après on va... tu auras le temps éventuellement de reprendre une autre position. Mais toi, qu'est-ce que tu avais mis déjà?

Cette interaction montre que ce ne sont pas les élèves, mais bien $\mathrm{M}$ qui régule à nouveau le déroulement des différents moments de la séquence. 


\section{Fonction de finalisation de l'étayage de l'enseignant}

Non seulement $\mathrm{M}$ séquentialise le déroulement de la séquence, mais son étayage remplit également une fonction de finalisation. Autrement dit, $\mathrm{M}$ oriente la discussion en fonction de la manière dont il a conçu le débat, et en fonction de savoirs préalablement étudiés qu'il vise à faire réinvestir par les élèves.

Dans la séquence de travail précédant le premier débat, les savoirs étudiés par les élèves relèvent de la situation locale des inondations à Brienz et d'éléments plus généraux en rapport au réchauffement climatique. Dans ce premier débat, pour que les élèves choisissent les propositions prioritaires à adresser aux autorités, $\mathrm{M}$ les fait voter à deux reprises. Il les fait voter une première fois, avant de les inviter à reprendre la parole et à défendre à nouveau leur point de vue. $M$ oriente manifestement les interventions des élèves pour que leur choix se porte sur une proposition locale et une proposition plus globale pour lutter contre le réchauffement climatique, de façon à faire ressurgir les savoirs étudiés dans les séquences préalables. M reprend en effet les propositions des élèves qui viennent d'être énoncées et souligne que selon lui, il y a deux positions importantes, la construction de barrages (une proposition avec un enjeu local) et un changement de mode de vie avec des conséquences sur l'usage de la voiture (une proposition avec un enjeu plus global):

(200) D'accord, mais là, on est à Brienz d'accord. Donc, il y a l'idée de proposer, plutôt que de construire des barrages, de construire des maisons qui seraient à chaque fois à reconstruire. Là, je dirais quill y a deux positions, si je résume. Il me semble: il y a ceux qui sont plutôt pour construire des barrages, je crois, et plutôt ceux qui sont pour changer de mode de vie, donc moins utiliser les vébicules. D'accord. Maintenant, je suis habitant à Brienz, OK, imaginez, j'habite là-bas, donc, vous proposez, vous, de changer de mode de vie, mais en quoi, comment ça va m'aider, pour les habitants, imaginez les habitants de Brienz. Pourquoi, est-ce que vous proposez ça, ceux qui sont, quatorze personnes qui sont pour changer le mode de vie, utiliser moins la voiture?

En résumant de cette manière, il semble que $\mathrm{M}$ opère une sélection des propositions énoncées par les élèves. Une élève avait en effet proposé par oral:

(20) Il faut qu'on achète moins, un peu moins des vêtements et des choses comme ça, parce que comme ça les usines fabriquent moins des choses et après ça pollue moins.

Dans cette proposition relativement développée, on peut relever une articulation entre une action individuelle et une action collective, deux types d'acteurs différents, et un raisonnement cause-conséquences. Pourtant, $M$ ne note pas cette proposition au tableau et comme il a été souligné plus haut, en remplissant une fonction de finalisation, son étayage le conduit à privilégier des propositions qui réinvestissent des savoirs préalablement étudiés.

Dans le deuxième débat, l'étayage de $\mathrm{M}$ remplit également une fonction de finalisation. Peut-être parce qu'il est influencé par la coutume didactique, $\mathrm{M}$ ne reprend pas dans ses interventions certains avis énoncés par les élèves mais privilé- 
gie un consensus. Dans la discussion, les avis des élèves sont partagés entre les projets $\mathrm{B}$ et $\mathrm{D}$ et très vite un dilemme se pose entre le financement de la recherche pour trouver des solutions à long terme contre le réchauffement climatique afin que de telles catastrophes ne se reproduisent plus, et la solidarité par l'aide d'urgence aux victimes d'inondations au Bangladesh. Lors de cette discussion, $M$ n'intervient que pour demander aux élèves s'ils sont tous d'accord, ou pour inciter ceux qui ne se sont pas exprimés à prendre la parole. Soudain, une élève se détache de ce dilemme en annonçant qu'elle a réparti la somme de 1000 francs de manière égale entre tous les projets, car selon elle,

(194) Ça peut tous [les] aider.

$\mathrm{M}$ ne tient pas compte de cette intervention, il passe la parole à un élève qui n'est pas d'accord et qui revient sur la défense du projet $D$. Plus loin, $M$ fait un point sur la discussion et c'est ici que l'on peut relever qu'il privilégie le consensus. En effet, il indique que les projets $\mathrm{B}$ et $\mathrm{D}$ sont les plus soutenus. Ce faisant, il ne donne pas de place au désaccord ou à un éventuel débat qui aurait pu naître de l'intervention de l'élève qui avait partagé la somme de manière égale entre les quatre projets. Le même scénario se reproduira une nouvelle fois. En effet, la discussion repart sur ce dilemme suscité par les projets B et D, avant qu'une autre élève annonce qu'elle pense qu'il faudrait répartir la somme entre tous les projets. A nouveau, $M$ ne réagit pas par rapport à cette proposition, et demande aux élèves qui ne se sont pas exprimés de le faire.

\section{Reformulation, modification du niveau de langage par l'enseignant}

Lorsque l'enseignant intervient pour recentrer l'objet de la discussion, le plus souvent il récapitule et reformule les propos des élèves en les paraphrasant. Ce faisant, il développe ce que vient de dire l'élève en y apportant un complément et en changeant parfois de niveau de formulation.

Dès le début du premier débat, lorsque $M$ annonce la consigne pour la séquence, il demande aux élèves ce qu'est une pétition. Un élève prend la parole:

(2) C'est quand on récolte des signatures. Pour pouvoir arrêter quelque chose qui se passe.

$M$ réplique de la manière suivante:

(3) Effectivement, il y a l'idée de récolter des avis, des signatures, on peut signer, et puis, pour donner un avis, demander quelque chose, tu as tout à fait raison.

Cette reformulation reprend la réponse de l'élève et la complète, puisque $\mathrm{M}$ y ajoute les idées qu'une pétition permet de donner un avis et de demander quelque chose.

Dans ce premier débat, l'enseignant reprend également les propositions des élèves par écrit, en les notant au tableau. Là encore, dans la reformulation des propositions, on peut constater que $\mathrm{M}$ développe les réponses des élèves. Oralement, un élève avait par exemple proposé:

(17) On peut aussi construire des maisons où quand les événements comme ça ils se passent, où il y a plus de risques, construire moins de maisons. 
La proposition retranscrite par $\mathrm{M}$ au tableau devient:

(103) Construire des maisons où il y a moins de risques, loin des lacs.

$\mathrm{M}$ a donc ajouté un élément de localisation, absent de la proposition de l'élève («loin des lacs»).

Dans le deuxième débat, un exemple permet par ailleurs de souligner que lorsque $\mathrm{M}$ reformule les propos des élèves, il opère parfois un changement de niveau de langage. Un élève argumente pour défendre le projet $\mathrm{D}$ de la manière suivante:

(198) Moi je suis d'accord pour heu, pour qu'on fasse des choses pour pas que ça se reproduise, mais il faut d'abord penser aux gens qui sont malades et puis les médicaments et tout.

$M$ reformule cette proposition en introduisant le terme d' «urgence», que l'élève n'avait pas mentionné:

(203) D'accord. Et ça tu penses... donc 1000 francs sur le D parce que pour toi c'est l'urgence qui compte. Alors on fait tous comme lui alors. Vous êtes d'accord?

Plus loin, $M$ soulignera à nouveau cette idée d'urgence, après l'intervention d'une autre élève:

(228) Je préfere donner 500 francs au projet D, pour aider les victimes et pour reconstruire, jusqu'à ce qu'ils trouvent pour heu, pour la science, jusqu'à ce qu'ils trouvent heu...le problème.

M: Si je comprends bien, tu as mis plus d'argent sur le D, en te disant que en attendant qu'on trouve quelque chose, il faut d'abord aider, et c'est ça qui est le plus important et le plus urgent, $\mathrm{mmmmhmmm}$. Bon.

L'introduction du terme «urgence» reflète un changement de niveau de langage opéré par M, il transforme le propos de l'élève en introduisant un terme plus élaboré. Il est possible d'interpréter ces compléments apportés par $\mathrm{M}$ ainsi que les changements de niveau de langage dans ses reformulations comme une manière lui permettant de se positionner dans son rôle d'enseignant dans le cadre du contrat didactique, et d'apporter dans la discussion des éléments qu'il vise à faire acquérir aux élèves.

Analyse spécifique des débats de la classe de secondaire Etant donné que, dans la classe secondaire, le deuxième débat a été animé par un élève, nous privilégions ici l'analyse de D1. L'organisation de l'analyse differe de celle menée pour la classe primaire. Nous avons privilégié l'analyse fine d'un extrait dans lequel l'intention de $\mathrm{M}$ a des effets sur le contenu des propos et produit des «simplifications-obstacles» à propos des notions de pouvoir et de cause (Audigier, 1998).

L'intention ou la finalisation que $M$ souhaite voir apparaître quant au contenu du débat est de placer ce dernier sur le terrain du développement durable. Dans la consigne écrite au tableau et commentée, en début de séquence, par $M$, le lien entre changements climatiques et inondations en Suisse est posé: «Etant 
donné qu'il y a des changements climatiques et que ces changements provoquent dans nos régions des situations climatiques extrêmes comme de très fortes précipitations, que faut-il faire pour qu'un événement comme celui de Brienz ne se reproduise plus?» Le débat proprement dit se découpe en trois moments: un premier (lignes 45 à 186) lors duquel les élèves «restent» essentiellement sur des considérations à propos des conséquences des inondations; un deuxième moment (lignes 187 à 270) dans lequel $\mathrm{M}$ tente d'orienter les élèves sur les causes et le lien avec les modifications du climat; un troisième moment (lignes 271 à 445) où il est effectué un choix parmi les propositions formulées, les mesures à proposer aux autorités. Nous présentons une analyse détaillée de deux épisodes du deuxième moment, lors duquel la volonté de finalisation de $\mathrm{M}$ s'exprime le plus explicitement.

\section{La question des "échelles du pouvoir»}

Dans un premier épisode (187-218), la distinction entre "population» et "autorité», fréquemment convoquée dans l'enseignement de l'histoire (Audigier, Aukkenthaler, Fink \& Haeberli 2002), est faite par une élève (Caroline ${ }^{14}$ ). La réaction de Caroline, la première dans le débat à ne pas être précédée par un tour de parole de $M$, fait suite aux propos tenus précédemment par un autre élève suggérant de sensibiliser la population aux risques liés aux situations climatiques extrêmes.

(189) Ca.: Ben, en fait on peut pas sensibili... enfin on peut sensib... (L'enseignant murmure: «Plus fort!»). On peut sensibiliser, mais cela sert à rien.

(191) M (en la coupant): Ça sert à rien pour toi?

(192) Ca (en continuant): C'est les auto-, c'est les autorités qu'on doit sensibiliser, parce que c'est eux qui peuvent faire quelque chose.

Plusieurs questions sont ici implicitement posées par la remarque de Caroline, notamment celle des prérogatives. Qui a le pouvoir de faire quoi? Cette question primordiale dans l'optique d'une adresse aux autorités des mesures à prendre pour lutter contre les changements climatiques, appelle une clarification de la question des «échelles de l'action». Si l'individu a certes un pouvoir, le pouvoir des autorités se situe à un autre niveau: il est le pouvoir institué doté des moyens de la collectivité. Entre ces deux pôles, une multitude d'acteurs entreprend des actions, à des niveaux intermédiaires, acteurs qu'il convient de distinguer.

Dans les réactions qui font suite à la remarque de Caroline, $\mathrm{M}$ n'oriente pas vraiment le débat vers un tel développement; il a plutôt hâte de finaliser celui-ci vers la question des causes. Il adopte, dans un premier temps, une posture dubitative quant à une absence de responsabilité de la "population» sur les "problèmes liés aux inondations».

(213) La population, elle peut rien faire? Qui peut réagir, qui aimerait réagir par rapport à cette... à cette dernière proposition? Est-ce que ici tout le monde considère que effectivement la population ne peut rien faire par rapport à ce problèmelà et que seules les autorités peuvent amener des solutions permettant de, de... limiter les risques d'inondations? Qui aimerait réagir? 
Les appels de $M$ resteront sans réponse sur le terrain des causes anthropiques des phénomènes climatiques étudiés.

\section{Les "vraies» causes}

L'enseignant fait alors appel plus directement à la notion de cause et tente de faire réagir un élève en particulier avant de solliciter l'ensemble de la classe:

(237) Gérard, a-t-on abordé les vraies causes? Heu... la population est-elle vraiment... pas vraiment responsable de ce qui se passe et ne pouvant pas vraiment faire quelque chose, c'est les autorités qui peuvent, est-ce que vous partagez cette opinion, oui, non, réagissez?

L'appel à la notion de causes, déjà présent plus tôt dans le débat (lignes 141 et 187) est ici renforcée par l'adjonction du terme "vrai», probablement pour souligner le fait que la bonne mesure n'a pas encore été évoquée, ni discutée. Il interpelle à nouveau la classe sur l'action possible des citoyens.

Les élèves qui interviennent ensuite reviennent sur des questions autres, liées notamment aux coûts d'une correction du fleuve. Dans les lignes qui suivent, $M$ revient à la charge en séquentialisant et en intégrant. Il fait appel aux souvenirs des élèves et au travail effectué préalablement à partir de la documentation:

(263) Réfléchissez peut-être par rapport aux causes que vous avez pu identifier, hein. Parce que si y a un problème et pis qu'on veut résoudre le problème, eh ben c'est généralement en s'attaquant aux causes du problème qu'on peut véritablement apporter des solutions au problème, hein. Donc, peut-être, une manière pour moi de vous relancer, réfléchissez peut-être sur les causes que vous avez pu identifier à travers la prise en connaissance de la documentation, pour voir quelles solutions on pourrait apporter. Oui.

Ce dernier tour de parole de $\mathrm{M}$ amène l'une des élèves (Monica) à proposer la mesure escomptée qui lie, causalement, réchauffement climatique et gaz à effet de serre:

(268) Mo: Mais les causes heu du réchauffement climatique, c'est y a beaucoup de heu de gaz à effet de serre, heu... non.

M: Comment tu formulerais ta proposition... par rapport à cette problém... problématique?

Mo: Diminuer les gaz à effet de serre.

M: Limiter les gaz à effet de serre, hein. Prendre des mesures... prendre des mesures pour limiter (il écrit au tableau) les émissions de gaz à effet de serre, voilà!

La séquence est en quelque sorte finalisée du point de vue du contenu: l'expression «voilà!» utilisée par $M$ le signifie de manière explicite, d'après nous. Pour en arriver là, $M$ s'est focalisé sur l'implication des élèves en tant que futurs citoyens et la recherche d'une cause. Cette centration a pour conséquence de réduire fortement la complexité de la chaîne causale dans les phénomènes liés au réchauffement climatique et des acteurs impliqués par les questions de développement durable. 


\section{Conclusion}

Arrivés au terme de cette présentation des résultats de l'analyse de quatre débats, nous reprenons nos principaux constats et les plaçons dans le cadre d'un questionnement sur les pratiques de débat en classe dans le domaine de l'éducation au développement durable et, plus largement, sur l'enseignement des questions socialement vives. Dans ce propos conclusif, trois thèmes questionnent le rôle de l'enseignant: la coutume scolaire, les développements thématiques, le sens commun.

Les pratiques de débat en classe sont réputées favoriser la construction de compétences sociales et argumentatives chez les élèves. À ce titre, tous les travaux insistent sur l'importance de confronter les paroles d'élèves entre elles (Dolz \& Schneuwly, 1998). Dans les débats analysés, la confrontation directe entre la parole des élèves est, la plupart du temps, médiatisée par une intervention enseignante. Ainsi, la transition de la parole par l'enseignant reste la règle. En l'espèce, les situations de débat proposées ne suffisent pas en elles-mêmes à couper certains des effets les plus puissants de la coutume scolaire. Au-delà de cette dernière question, la persistance de la médiation enseignante dans l'échange interroge le rôle et la place de l'enseignant dans les débats, entre garant de la "vérité» des débats et facilitateur des échanges (Balacheff, 1988).

Du point de vue de l'étayage (Bruner, 1983/1996), les propos enseignants dénotent une fonction de finalisation des débats et de délimitation des frontières entre les unités thématiques; les incitations à poursuivre au sein d'un développement thématique sont, quant à elles, beaucoup plus rares ${ }^{15}$. La discrétion de la fonction de développement dans les propos enseignants s'explique, en partie, par les contraintes liées à la forme scolaire (Vincent, 1994) qui encadrent l'action de l'enseignant et auxquelles les situations de débat n'échappent évidemment pas. Le souci de faire avancer les opérations et d'achever les débats qui se déroulaient, chacun, sur une seule période ${ }^{16}$, est probablement un facteur explicatif de la prégnance des fonctions de finalisation dans les propos enseignants. Au-delà des processus de justification, une mise en œuvre de la fonction de développement a la vertu de complexifier les propos et de donner l'occasion, pour les autres participants au débat, de confronter les propos exprimés.

Par ailleurs, les analyses ont mis en lumière un processus de sélection et de transformation des propos des élèves par les enseignants. Ces derniers complètent, modifient et placent ce que disent les élèves dans des univers sémantiques qui ne sont pas nécessairement les mêmes que ceux des élèves. Or, ces déplacements sémantiques se font, la plupart du temps, de manière tacite et implicite, sans qu'il y ait un arrêt ou un retour sur ces opérations de transformation du langage. Ces processus largement non conscients demanderaient à être explicités, notamment dans l'optique d'identifier les «entrées disciplinaires» sur l'objet du débat et, in fine, d'introduire les élèves aux différentes manières que les disciplines ont de faire des mondes (Audigier, 1993). 
Enfin, les analyses des extraits dans lesquels les notions de "cause» et de "pouvoir» sont abordées, explicitent, dans les propos enseignants, ce que Hewstone et Moscovici nomment la "pensée binaire ou la persistance des modes de pensée enfantins» dans les formes de raisonnement de la pensée du sens commun (Hewstone \& Moscovici, 1984). La simplification opérée sur la question des «échelles du pouvoir», opposant le pouvoir des individus à celui des autorités sans prendre en compte la multiplicité des acteurs intermédiaires, illustre la logique de réduction de la complexité qui préside au «jeu du sens commun». Selon ces approches, le sens commun, ou les savoirs du quotidien selon les qualifications que l'on utilise, ne sont pas pensés comme des obstacles ou des erreurs qu'il s'agirait de franchir ou d'éradiquer, mais comme les composantes d'une pensée personnelle et sociale qu'il s'agit de raisonner et d'enrichir.

\section{Notes}

1 Sur ce thème, voir Simonneaux \& Legardez (2006).

2 Le premier volet de la recherche a débuté en septembre 2007 et s'est achevé en mars 2009; le deuxième volet d'une durée de trente mois a débuté le $1^{\text {er }}$ septembre 2009.

3 Pour plus de détails sur les membres et les activités de l'ERDESS: www.unige.ch/fapse/didactsciensoc

4 Les projets en question peuvent être résumés comme suit: (A) limiter les rejets de gaz à effet de serre dans le domaine des transports; (B) aider la recherche scientifique pour développer des techniques non polluantes; (C) lutter pour la conservation d'emplois en Suisse dans le secteur touristique touché par le manque de neige; (D) aide d'urgence aux victimes des inondations au Bangladesh.

5 Dès les premiers degrés scolaires, les élèves apprennent à adopter certains comportements avant de prendre la parole et à attendre l'autorisation du maître pour ce faire.

6 De ce point de vue, la situation de débat en classe est clairement amenée à modifier la forme scolaire des relations sociales et de transmission des savoirs qui prévaut depuis le XVIe siècle dans les sociétés européennes (Vincent, 1994).

7 L'identification des protagonistes et le comptage des interventions de chacun d'entre eux ont été réalisés au moyen du logiciel Transana, qui permet, entre autres fonctions, la transcription écrite des enregistrements audio-visuels des débats.

8 Nous avons utilisé, à partir des transcriptions, la fonction «statistiques» dans le logiciel Word.

9 Ces chiffres comprennent tous les différents moments des débats, donc également la présentation du débat par l'animateur.

10 Par «protagonistes», nous entendons les participants actifs aux débats, c'est-à-dire prenant la parole, à l'exclusion de l'animateur.

11 Par «intervention», nous entendons les tours de parole qui peuvent, selon les cas, compter de un (par exemple, donner la parole à un élève qui lève la main en l'appelant par son prénom) à plusieurs dizaines de mots (par exemple, présenter le déroulement du débat).

12 Dans le débat C2_D2 animé par l'élève, l'enseignant est en position d'observateur. Il ne prononce aucun mot.

13 Les numéros entre parenthèses sont indiqués à titre de repères; ils correspondent à la numérotation des lignes de la transcription.

14 Tous les prénoms désignant des élèves sont fictifs.

15 Cette discrétion de la fonction de développement se retrouve, à l'exception notable d'un cas, dans les autres classes qui ont participé à l'enquête.

16 Une période correspond à une leçon de 45 ou de 50 minutes. 
Bibliographie

Audigier, F. (1991). Enseigner la société, transmettre des valeurs. Revue française de pédagogie, 94, 37-48.

Audigier, F. (1993). Les représentations que les élèves ont de l'histoire et de la géographie. À la recherche des modèles disciplinaires, entre leur définition par l'institution et leur appropriation par les élèves. Thèse pour le Doctorat d'État, Université de Paris VII.

Audigier, F. (Éd.). (1998). Contributions à l'étude de la causalité et des productions des élèves dans l'enseignement de l'histoire et de la géographie. Paris: INRP.

Audigier, F., Auckenthaler, Y., Fink, N. \& Haeberli, P. (2002). Leçons d'histoire à l'école primaire. Cartable de Clio, 2, 194-217.

Balacheff, N. (1988). Le contrat et la coutume, deux registres des interactions didactiques. In C. Laborde (Éd.), Actes du premier colloque franco-allemand de didactique des mathématiques et de l'informatique (Lumigny) (pp. 15-28). Grenoble: La Pensée sauvage.

Bruner, J. (1983/1996). Le développement de l'enfant. Savoir faire, savoir dire. Paris: PUF.

Dolz, J. \& Schneuwly, B. (1998). Pour un enseignement de l'oral: Initiation aux genres formels à l'école. Paris: ESF.

Grize, J.-B. (1996). Logique naturelle et communication. Paris: PUF.

Hewstone, M. \& Moscovici, S. (1984). De la science au sens commun. In S. Moscovici (Éd.), Psychologie sociale (pp. 539-571). Paris: PUF.

Lenoir, Y. \& Sauvé, L. (1998). De l'interdisciplinarité scolaire à l'interdisciplinarité dans la formation à l'enseignement: Un état de la question. Revue française de pédagogie, 124, 121 153.

Nonnon, E. (1997). Quels outils se donner pour lire la dynamique des interactions et le travail sur les contenus de discours? Enjeux, 39-40, 12-49.

Simonneaux, L. \& Legardez, A. (Éd.). (2006). L'école à l'épreuve de l'actualité. Enseigner les questions vives. Paris: ESF.

Vincent, G. (Éd.). (1994). L'éducation prisonnière de la forme scolaire? Scolarisation et socialisation dans les sociétés industrielles. Lyon: Presses Universitaires de Lyon.

Mots clés: Débat, développement durable, enseignant, didactique, coutume didactique

\section{Die Rolle der Lehrperson in Gesprächssituationen zu Themen der Bildung für nachhaltige Entwicklung}

\section{Zusammenfassung}

Die nachfolgend präsentierte Studie des Forschungsteams für Didaktik und Epistemologie in Sozialwissenschaften (ERDESS) der Universität Genf beschäftigt sich spezifisch mit der Situation und Rolle der Lehrperson in Debatten. Die Studie ist Bestandteil einer von ERDESS an Schulen der französischsprachigen Schweiz durchgeführten Untersuchung. Bezogen auf die Debatten interessieren sich die Autoren und Autorinnen für die Prägungskraft von Routinen und schulischen Zwängen - sowohl im Hinblick auf die Dynamik des Gesprächs, als auch im Hinblick auf die Gesprächsinhalte. Die Analyse von vier jeweils in einer Primar- und einer Sekundarklasse durchgeführten Debatten zeigt, dass Spannungen, die den Klassenbetrieb charakterisieren, durchaus einen Einfluss auf die De- 
batten zeitigen. Die Rolle der Lehrperson wurde insbesondere mittels einer dreifachen Unterscheidung der ihr zugeschriebenen Gesprächsregulierungsfunktion analysiert.

Schlagworte: Debatte, nachhaltige Entwicklung, Lehrperson, Didaktik, Unterrichtsroutine

\section{II ruolo dell'insegnante in situazioni di discussione sullo sviluppo sostenibile}

\section{Riassunto}

Questo testo è parte di una ricerca condotta nelle scuole della Svizzera francese dal gruppo di ricerca in didattica ed epistemologia delle scienze sociali (ERDESS) dell'Università di Ginevra. Si occupa in particolare del ruolo del docente in situazioni di discussione. L'articolo pone attenzione sull'influenza di abitudini e condizionamenti scolastici, non solo in termini di dinamica di interazione, ma anche in termini di contenuti degli scambi. Le analisi di quattro dibattiti in due classi, una della scuola primaria e una della scuola secondaria, mostrano che le situazioni di discussione non sono immuni alle tensioni che caratterizzano l'insegnamento scolastico. Una triplice distinzione viene usata per analizzare il ruolo del docente relativamente alla moderazione degli scambi.

Parole chiave: discussione, sviluppo sostenibile, docente, didattica, abitudini scolastiche

\section{The role of the teacher in debate situations on sustainable development education themes}

\section{Abstract}

This text is part of a research conducted in French speaking Swiss schools by the Research Team in Didactics and Epistemology of Social Sciences (ERDESS) at the University of Geneva. It specifically deals with the place and role of the teacher in situations of debate. The point of interest is the influence of habits and school constraints not only in terms of dynamics of interactions but also in terms of content of the exchanges. The analyses of four debates in two classes, one in the primary level, one in the secondary level, show that situations of debate are not immune to the tensions that characterize the school teaching. A triple distinction is used to analyse the role of the teacher regarding the exchanges regulation function that he's led to endorse.

Key words: Debate, sustainable development, teacher, didactic, school habits 